\title{
Piecewise Adaptive Sliding Mode Control for Aeroengine Networked Control Systems with Resource Constraints
}

\author{
Bin Zhou $\mathbb{D}^{1},{ }^{1}$ Shousheng Xie, ${ }^{1}$ Litong Ren, ${ }^{2}$ Lei Wang, ${ }^{1}$ Yu Zhang $\mathbb{D},{ }^{1}$ \\ Ledi Zhang, ${ }^{1}$ and Hao Wang ${ }^{1}$ \\ ${ }^{1}$ Aeronautics and Astronautics Engineering College, Air Force Engineering University, Xian, China \\ ${ }^{2} 94314$ Unit, Air Force of Chinese People's Liberation Army, Zhengzhou, China \\ Correspondence should be addressed to Bin Zhou; icy_sky@foxmail.com
}

Received 11 June 2018; Revised 15 November 2018; Accepted 12 February 2019; Published 26 February 2019

Academic Editor: Basil M. Al-Hadithi

Copyright (C) 2019 Bin Zhou et al. This is an open access article distributed under the Creative Commons Attribution License, which permits unrestricted use, distribution, and reproduction in any medium, provided the original work is properly cited.

\begin{abstract}
Within the future application of wireless network for the aeroengine control problem, resource constraints (caused by the limitation of hardware) and network traffic restriction must be considered as one of the difficulties to be solved; thus, the network connection and transmission efficiency can be ensured. With focus on the problem of active packed dropout, a MEF-TOD (Maximum Error First-Try Once Discard) scheduling based network parameter and sliding mode joint design method has been proposed. First, a scheduling protocol strained control system and network parameter joint model have been established based on MEF-TOD scheduling strategy, taking sampling period and data packet capacity as unknown network parameters. Subsequently, considering the influence of scheduling strategy, a sliding surface containing a compensation term has been designed, and then a sliding mode parameter and unknown network parameter heuristic joint design method has been developed. Finally, an attenuation factor based piecewise adaptive sliding mode strategy has been designed considering the influence of sampling period on system performance. Simulation results indicate that the joint design method can obtain the network parameter group which has the minimum performance function upper bound, thus achieving relatively high network utilization. The proposed piecewise adaptive sliding mode controller has good dynamic performance and is robust to the packet dropout problem caused by network scheduling and can effectively suppress chattering.
\end{abstract}

\section{Introduction}

Modern aircraft are expected to be tolerant of complicated flight condition and to work throughout more signal communication, the result of which is that the system shows more complexity. Within the future application of wireless network for the aeroengine control problem, resource constraints (caused by the limitation of hardware) and network traffic restriction must be considered as one of the difficulties to be solved; thus, the network connection and transmission efficiency can be ensured. Sliding mode control, a reliable and tried-and-true method, is developed for the complex environment, which could be the theoretical support for designing and testing aeroengine networked control system in engineering practice.

The main feature and advantage of networked control systems is the information transmission technology based on a bus network [1]. However, bandwidth of bus networks is limited because of the limitations imposed by the level of industrial design and system working conditions. When a large number of intelligent nodes such as sensors and actuators are present, only some nodes can be permitted to access the network during an access period, which influences the real-time transmission of sensor and controller information [2]. Furthermore, some high priority signals (security warnings, control interrupts, etc.) should be set in control systems, and these are not transmitted in fixed periods. Once activated, the real-time network resources will be occupied [3], causing uncertain data packet dropout and longer transmission delays.

Resource constraint problems for networked control systems include media access restricted, bits per second constraints, and message transmission rate constraints. Other than in the traditional concentrated systems, media access 
restricted indicates that only access requirements of a limited number of network nodes can be satisfied at the same time. Because of the node access conflicts, it is of great importance to introduce network scheduling for networked control systems to clear and define node access rules based on reasonable network resources distribution in accordance with mission requirements [4-6] by applying network scheduling algorithms like the Rate Monotonic (RM) algorithm, Earliest Deadline First (EDF) algorithm, MEF-TOD algorithm, etc. These algorithms improve Quality of Performance (QoP) of the systems by perfecting Quality of Service (QoS) of the networks on the premise of guaranteeing system properties. From [7], when network bandwidth is limited, QoP is incompatible with QoS. If sampling period is longer and data packet capacity is lower, more data messages must be abandoned for better QoS, which deteriorates QoP.

Time delay and packet dropout have been researched for network systems in various studies. However, networks in these references are simplified by using serial communications, which means that signals are transmitted based on the sequence of a receiving terminal, and network bandwidth and instantaneous load are considered for time delay and packet dropout only as uncertain random parameters with upper bounds. In [8], a method modeling a time delay system is adopted, which combines the network induced delay and packet dropout as a Markov random time delay to analyze system stability and design the controller. As network bandwidth is limited, packet dropout takes on a new meaning in network systems. When nodes fail to access the network, their message will be abandoned in the current period, and thus packet dropout occurs, which has features as follows: (1) because network scheduling protocols are adopted, if some node fails in competition and its packet is dropped at sampling time $k$, higher priority will always be given to the node at sampling time $k+1$, so packet dropout is discontinuous for one node; (2) as network scheduling protocols have some specific significance, message loss is known to the controller, and it will not be influenced by network load. Given the above, packet dropout cannot be simplified as an uncertain random time delay.

So in the condition of controllable packet dropout, it is important that controller and scheduling strategy are jointly designed to obtain the best performance for faulttolerant control of networked control systems with resource constraints. In recent years, researchers have paid more attention to the resource constraints problem. The authors in [9] proposed a joint design method with TOD dynamic scheduling strategy and a robust $H_{\infty}$ controller; however, the scheduling of control signals was not considered in the illustrative example. In [10], a new scheduling strategy was proposed, which depends on average dwell time for similar networked control systems, and a discrete-time switched system with parameter uncertainty was modeled, in which the controller is collaboratively designed based on multiLyapunov functions. However, the influence of the sampling period on control quality was not researched. The authors in [11] focused on a joint design of TOD scheduling strategy and quantification control, and the closed-loop system was optimized; however, the structure is not suitable for an aeroengine central control unit and Controller Area Network (CAN) bus because the control signals are not transmitted through the network. The studies above provide new ideas for networked control systems with resource constraints, which belong to the design category of fixed network parameters. The authors in [12] derived the optimal offline schedule for a communication sequence that is allowed to be timevarying; however, the methods should be verified in a real Multiple-Input Multiple-Output (MIMO) system. In fact, a strongly coupled relationship exists between network and controller performances, and although [13] proposed an optimal codesign method, the scheduling process and the state feedback controller performance under different network conditions should be deeply analyzed. The authors in [14] employed a Lyapunov-Metzler inequalities method for the simultaneous design problem of dynamic scheduling and feedback control; however, the network parameters were not included in their design. In [15], a detailed report on the influence of network parameters (sampling period $T$, data packet capacity $P$, etc.) on discrete-time sliding mode control chattering was presented; however, the codesign of the network and sliding mode controller should also be investigated. More recently, [16] designed an adaptive sliding mode controller for a complex system, but resource constraints should also be considered after the presence of time delays, external disturbances, and sector nonlinearity of inputs. The authors in [17] investigated sliding mode control combined with sampling rate control for networked control systems; however, the packet disordering research conducted still belongs to passive packet dropout. In [18], a codesign scheme of a hybrid scheduling strategy and the improved $\mathrm{H}$ infinity output feedback controller was proposed; however, more complex conditions should be considered because they assumed that only one node can access the network at most at any time instant. In [19], a deadband scheduling method was proposed for a wireless networked control system, but owing to the fixed priority, the static scheduling used cannot ensure system stability and robustness in an uncertain open environment. The authors in [20] suggested a scheduling method in which the state update time was adjusted according to the real-time network congestion situation, but the strategy as to which data packet should be transmitted was not considered. Thus, in summary, it is necessary to design controllers with network parameters in consideration of (1) the scheduling of both sensor and control signals; (2) the influence of the sampling period on QoS; (3) MIMO systems; (4) the active packet dropout; and (5) dynamic scheduling with more than one node accessible in the bus network.

Focusing on the active packet dropout problem in scheduling strategy and robust performance of sliding mode control, this paper proposes a new joint design method based on MEF-TOD network parameters and sliding mode control. First, for the MEF-TOD scheduling strategy, the influence of active packet dropout on system performance is analyzed, and transmissions of state and control messages with resource constraints are modeled. Second, based on the idea of joint design of the controller, sampling period $T$ and data packet capacity $P$ are chosen as unknown network parameters, and the joint model control system including network parameters 
with scheduling protocol constraints is constructed. Third, in consideration of scheduling strategy influence, a discrete sliding plane with data compensation is designed, and the heuristic joint design steps for ensuring optimal performance and sliding mode convergence are developed. Finally, in consideration of the influence of sampling period to system performance, a piecewise adaptive sliding mode control strategy is designed based on attenuation factors, which can ensure system accessibility and effectively reduce chattering of the quasi-sliding mode.

\section{Joint Modeling of Aeroengine Networked Control Systems with Resource Constraints}

2.1. System Description. Figure 1 shows the structure of aeroengine networked control systems with resource constraints, in which the intelligent actuators, intelligent sensors, and controllers transfer information through a public bus network. As the system employs MEF-TOD scheduling strategy [21], node access permissions would be reassigned for better QoS when the system state and control variables exceed the node access limitation in the network.

With system time delay and network parameters influences considered, the control system can be descripted as

$$
\begin{aligned}
& \dot{x}(t)=f(x(t), u(t), T, P) \\
& x(t)=\varphi(t), \quad t \in\left[-\tau_{0}, 0\right]
\end{aligned}
$$

Here, $x(t) \in R^{n}$ is a state variable, $u(t) \in R^{m}$ is a control variable, $\tau_{0}$ is the time delay of the network system at time zero, and $\varphi(t)$ is the initial system state function. $T$ is the sampling period, $P$ is the data packet capacity, which is transferred during one period, and both are uncertain constant network parameters. Furthermore, network bandwidth $\eta$ and nonreal-time data width $\theta_{k}$ are also network parameters related to scheduling, which are preset in this paper.

For easier derivation, assumptions pertaining to the system shown in Figure 1 are made as follows.

Assumption 1. A time-driven mode is adopted for sensor nodes, and an event-driven mode is adopted for controller and actuator nodes.

Assumption 2. Node data is transferred in one packet, and there is no wrong order condition.

Assumption 3. Random delays $\tau_{s c}$ and $\tau_{c a}$ occur during the transmission of sensor-to-controller and controller-toactuator, respectively. In the scheduling strategy, $\tau=\tau_{s c}+$ $\tau_{c a}<T$.

As media access is always constrained in the actual system, only $d_{s}\left(0<d_{s} \leq n\right)$ state data and $d_{c}\left(0<d_{c} \leq m\right)$ control data can be transferred by the bus network during one sample period, and the network accessibility of every node is decided by the network scheduling algorithm. The MEF-TOD scheduling algorithm uses system real-time information to dynamically allocate resources and includes two parts: (1) MEF scheduling strategy, which calculates the weighted sum of errors between the previous data and the current data transferred by each node, and higher access permissions will be assigned to the nodes with larger errors; (2) TOD distribution protocol, which abandons the data packets of nodes if they fail when competing for network resources, and new sample data will be adopted in order to keep node data real-time in the next competition.

For the MEF-TOD strategy, sensor-controller scheduling variable $\rho(k)$ and controller-actuator scheduling variable $\delta(k)$ are introduced as

$$
\begin{aligned}
& \rho_{i}(k)= \begin{cases}1 & x_{i}(k) \text { successfully transferred } \\
0 & \text { otherwise }\end{cases} \\
& i=1,2, \ldots, n \\
& \delta_{j}(k)= \begin{cases}1 & u_{j}(k) \text { successfully transferred } \\
0 & \text { otherwise }\end{cases} \\
& j=1,2, \ldots, m
\end{aligned}
$$

At time $k$, data of sensors and actuators which pass the competition transfer their data to network receivers in order to update data, and node data which have not been updated will retain the values in the last period through a zero-order holder $(\mathrm{ZOH})$. Here, scheduling matrices of sensor nodes are defined as

$$
\Gamma(k)=\operatorname{diag}\left(\rho_{1}(k), \rho_{2}(k), \cdots, \rho_{n}(k)\right)
$$

So the state values received by the controller after scheduling are

$$
\omega(k)=\Gamma_{\sigma}(k) x(k)+\left(I-\Gamma_{\sigma}(k)\right) \omega(k-1)
$$

In the same way, scheduling matrices of actuator nodes are defined as

$$
\Delta(k)=\operatorname{diag}\left(\delta_{1}(k), \delta_{2}(k), \cdots, \delta_{m}(k)\right)
$$

and the control values received by actuators after scheduling are

$$
v(k)=\Delta_{\sigma}(k) u(k)+\left(I-\Delta_{\sigma}(k)\right) v(k-1)
$$

Under the condition of medium access constraints, the quantity of nodes which access the network at the same time should satisfy $d_{s}(k)+d_{c}(k) \leq P$, so there will be $C_{n+m}^{P}=(n+m) ! /[(n+m-P) ! P !]=N$ data transmission patterns in the current network environment, and the corresponding scheduling matrix is $\Psi_{i}(k)=\operatorname{diag}(\Gamma(k), \Delta(k))(i=$ $1,2, \cdots, N)$. 


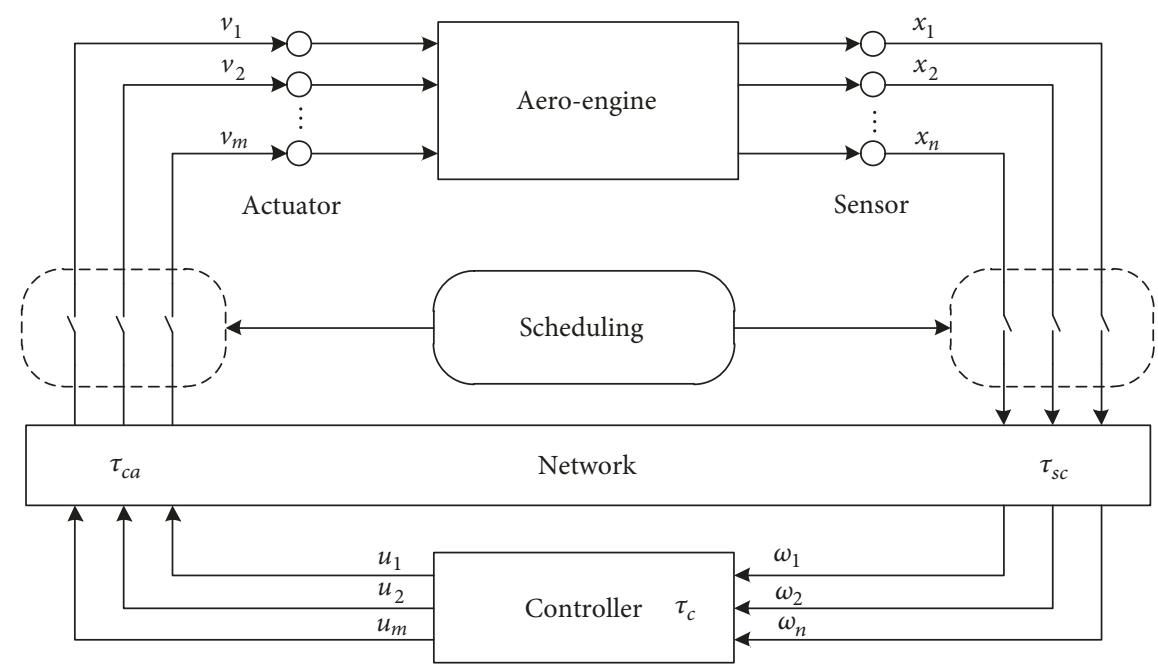

FIGURE 1: Structure of aeroengine networked control systems with resource constraints.

For the MEF-TOD scheduling protocol, the node data weighted error $\bar{e}(k)$ at the current instant should be obtained first and is defined as

$$
\begin{aligned}
\bar{e}(k) & =\left[\begin{array}{c}
e^{x}(k) \\
e^{u}(k)
\end{array}\right]=\left(\frac{x_{1}(k)-x_{1}(k-1)}{x_{1}(k-1)},\right. \\
& \ldots \frac{x_{n}(k)-x_{n}(k-1)}{x_{n}(k-1)}, \frac{u_{1}(k-1)-u_{1}(k-2)}{u_{1}(k-2)}, \\
& \left.\ldots \frac{u_{m}(k-1)-u_{m}(k-2)}{u_{m}(k-2)}\right)^{T}
\end{aligned}
$$

According to the TOD distribution protocol, the switching function of transmission pattern is defined as

$$
\sigma=\arg \max \left\{\left\|\Psi_{1}(k) \bar{e}_{1}(k)\right\|, \cdots,\left\|\Psi_{N}(k) \bar{e}_{N}(k)\right\|\right\}
$$

where $\arg$ is the function used to obtain subscripts, $\Psi_{i}(i=$ $1,2, \ldots, N)$ is the corresponding $i$-th transmission pattern, and $\sigma \in\{1,2, \ldots, N\}$.

2.2. Joint Design Model for Network and Control System Parameters. With resource constraints, to reduce the conservatism of the control system and increase utilization of network resources, the controller will be designed by considering network parameters. According to the presentation in the last section, sampling time $T$ and data packet capacity $P$ are considered as undetermined network parameters, while network bandwidth $\eta$ and non-real-time data width $\theta_{k}$ are considered as known quantities. So the joint model of network parameters and control system parameters will be designed by the conversion relationship between the parameters and system time delay.

Ignoring noise, the continuous model of the aeroengine networked control system is established as

$$
\begin{aligned}
& \dot{x}(t)=A_{c} x(t)+B_{c} u(t) \\
& y(t)=C_{c} x(t)
\end{aligned}
$$

From [13], under the condition of network scheduling capacity and no data packet dropout, the following relationship holds.

$$
(k+1) \bar{P}+\bar{\theta}_{k}=(k T+\tau(k)) \eta
$$

Here, $\bar{P}=\iota P, \iota$ is data width of a single transmission variable, and $\bar{\theta}_{k}=\sum_{i=0}^{k} \theta_{i}$. So the following expression can be obtained.

$$
\tau(k)=\frac{(k+1) \bar{P}+\bar{\theta}_{k}}{\eta}-k T
$$

Based on Assumptions 1-3, a joint model considering network parameters and control system parameters can be established by system (9) as

$$
\begin{aligned}
x(k+1)= & A x(k)+\left(B_{0}+D F_{k} E\right) v(k) \\
& +\left(B_{1}-D F_{k} E\right) v(k-1) \\
y(k)= & C x(k)
\end{aligned}
$$

where

$$
\begin{aligned}
A & =e^{A_{c} T}, \\
B_{0} & =\int_{0}^{(2 T \eta-\bar{P}) / \eta} e^{A_{c} s} d s \cdot B, \\
B_{1} & =e^{A_{c}((2 T \eta-\bar{P}) / \eta)} \int_{0}^{(\bar{P}-T \eta) / \eta} e^{A_{c} s} d s \cdot B, \\
D & =\beta e^{A_{c}((2 T \eta-\bar{P}) / \eta)}, \\
F_{k} & =\beta^{-1} \int_{0}^{-\left(k \bar{P}^{2}+\bar{\theta}_{k}-(k-1) T \eta\right) / \eta} e^{A_{c} s} d s, \\
E & =B, \\
C & =C_{c} .
\end{aligned}
$$


When system maximal sampling period $T_{\max }$ and non-realtime data maximal allowable width $\theta_{k \max }$ are defined, $\beta=$ $\max _{\tau(k-1)}\left\|\int_{0}^{-\tau(k-1)-\theta_{k \max }} e^{A_{c} s} d s\right\|_{2}=\left\|\int_{0}^{T_{\max }+\theta_{k \max }} e^{A_{c} s} d s\right\|_{2}$ and $F_{k}^{T} F_{k} \leq I$.

To obtain a better expression, $H=e^{A_{c}((2 T \eta-\bar{P}) / \eta)}$ is defined, and when matrix $A_{c}$ is invertible,

$$
\begin{aligned}
& B_{0}=A_{c}^{-1}(H-I), \\
& B_{1}=A_{c}^{-1}(A-H), \\
& D=\beta H
\end{aligned}
$$

Eq. (14) is substituted into (12), and $\Delta B=\beta H F_{k} E$ is defined, and the following joint model can be obtained.

$$
\begin{aligned}
x(k+1)= & A x(k)+\left(B_{0}+\Delta B\right) v(k) \\
& +\left(B_{1}-\Delta B\right) v(k-1) \\
y(k)= & C x(k) \\
\omega(k)= & \Gamma_{\sigma}(k) x(k)+\left(I-\Gamma_{\sigma}(k)\right) \omega(k-1) \\
v(k)= & \Delta_{\sigma}(k) u(k)+\left(I-\Delta_{\sigma}(k)\right) v(k-1)
\end{aligned}
$$

When $M_{0}=B_{0}+\Delta B$ and $M_{1}=B_{1}-\Delta B$, the model can be improved as

$$
\begin{aligned}
x(k+1)= & A x(k)+M_{0} \Delta_{\sigma} u(k) \\
& +\left(M_{0}\left(I-\Delta_{\sigma}\right)+M_{1}\right) v(k-1) \\
y(k)= & C x(k) \\
\omega(k)= & \Gamma_{\sigma} x(k)+\left(I-\Gamma_{\sigma}\right) \omega(k-1) \\
v(k)= & \Delta_{\sigma} u(k)+\left(I-\Delta_{\sigma}\right) v(k-1)
\end{aligned}
$$

\section{Joint Design of Sliding Mode Surface Gain and Network Parameters}

During the design process of a networked control system with resource constraints, both QoS and QoP should be considered, so proper network parameters should be adopted to provide the optimal control system performance under the limited network bandwidth condition. In this section, the joint model (16) is adopted as the controlled object, and a guaranteed cost joint control method is proposed based on a sliding mode controller. Aiming at cost function $J$, sliding mode surface parameters are designed for the system sliding mode dynamics to be asymptotically stable and with a cost function upper bound of $J^{*}$.

3.1. Linear Sliding Mode Surface Design considering Network Scheduling. The nominal form of system (16) is

$$
\begin{aligned}
x(k+1)= & A x(k)+B_{0} \Delta_{\sigma} u(k) \\
& +\left(B_{0}\left(I-\Delta_{\sigma}\right)+B_{1}\right) v(k-1)
\end{aligned}
$$

When building the discrete sliding mode controller, the influence of the scheduling strategy should be considered, so the discrete sliding mode surface can be defined as follows:

$$
\begin{aligned}
s(k)= & L_{\sigma} \omega(k)-\omega(k) \\
\omega(k)= & L_{\sigma}\left(B_{0} K_{\sigma}+\left(I-\Gamma_{\sigma}\right)\right) \omega(k-1) \\
& -L_{\sigma}\left(\Gamma_{\sigma} B_{0}\left(I-\Delta_{\sigma}\right)+\left(I-\Gamma_{\sigma}\right) B_{0}\right) u(k-1) \\
& +L_{\sigma} \Gamma_{\sigma}\left(B_{0}\left(I-\Delta_{\sigma}\right)+B_{1}\right) v(k-2)
\end{aligned}
$$

where sliding mode surface gain $L_{\sigma} \in R^{m \times n}$ should satisfy $L_{\sigma} B_{0}$ nonsingularly, and matrix $K_{\sigma} \in R^{m \times n}$ is the gain matrix which should be designed.

From (18),

$$
\begin{aligned}
s(k+1)= & L_{\sigma} \omega(k+1)-\omega(k+1) \\
\omega(k+1)= & L_{\sigma}\left(B_{0} K_{\sigma}+\left(I-\Gamma_{\sigma}\right)\right) \omega(k) \\
& -L_{\sigma}\left(\Gamma_{\sigma} B_{0}\left(I-\Delta_{\sigma}\right)+\left(I-\Gamma_{\sigma}\right) B_{0}\right) u(k) \\
& +L_{\sigma} \Gamma_{\sigma}\left(B_{0}\left(I-\Delta_{\sigma}\right)+B_{1}\right) v(k-1)
\end{aligned}
$$

When $s(k+1)=0$, the equivalent control law can be obtained as follows:

$$
\begin{aligned}
u_{e q}(k)= & \left(K_{\sigma} \Gamma_{\sigma}-\left(L_{\sigma} B_{0}\right)^{-1} L_{\sigma} \Gamma_{\sigma} A\right) x(k) \\
& +K_{\sigma}\left(I-\Gamma_{\sigma}\right) \omega(k-1)
\end{aligned}
$$

By substituting (20) into (16), the dynamic equation of the system sliding mode motion can be defined as

$$
\begin{aligned}
& x(k+1) \\
& =\left(A+M_{0} \Delta_{\sigma} K_{\sigma} \Gamma_{\sigma}-M_{0} \Delta_{\sigma}\left(L_{\sigma} B_{0}\right)^{-1} L_{\sigma} \Gamma_{\sigma} A\right) x(k) \\
& +M_{0} \Delta_{\sigma} K_{\sigma}\left(I-\Gamma_{\sigma}\right) \omega(k-1) \\
& +\left(M_{0}\left(I-\Delta_{\sigma}\right)+M_{1}\right) v(k-1) \\
& \omega(k)=\Gamma_{\sigma} x(k)+\left(I-\Gamma_{\sigma}\right) \omega(k-1) \\
& v(k) \\
& =\Delta_{\sigma}\left(K_{\sigma} \Gamma_{\sigma}-\left(L_{\sigma} B_{0}\right)^{-1} L_{\sigma} \Gamma_{\sigma} A\right) x(k) \\
& +\Delta_{\sigma} K_{\sigma}\left(I-\Gamma_{\sigma}\right) \omega(k-1)+\left(I-\Delta_{\sigma}\right) v(k-1)
\end{aligned}
$$

3.2. Sliding Mode Motion Stability Analysis. In consideration of the influence of time delay on system performance, Lemma 4 is provided for changing the time delay uncertainty into the product of certain matrices in some interval, in order to facilitate the robustness discussion of sliding mode motion.

Lemma 4 (see [22]). Suppose that $Y, M$, and $N$ are matrices with proper dimensions, and $Y$ is symmetric, so when $F(k)$ 
satisfies $F^{T}(k) F(k) \leq I$, the following relationship is workable.

$$
Y+M F(k) N+N^{T} F^{T}(k) M^{T}<0
$$

$Y+\varepsilon^{-1} N^{T} N+\varepsilon M M^{T}<0$ if and only if there is a scalar $\varepsilon>0$.

Furthermore, for a networked control system with resource constraints, network parameters and controller performance should be considered in the design process of the controller. By designing a cost function, the existence of an upper bound of the system control cost can be ensured, and the performance degradation of the system and the excessive waste of controller resource can be avoided.

The cost function is defined as

$$
J=\sum_{k=0}^{\infty} x(k)^{T} R_{1} x(k)+u(k)^{T} R_{2} u(k)
$$

where $R_{1}, R_{2}$ are known weighting matrices of the cost function, which is positive definite. In the equation, the first item represents the total system energy, and the second term represents the system consumption. The basic goal of designing a guaranteed cost controller is ensuring the existence of the upper bound $J^{*}$ in function $J$.

Theorem 5. For the networked control system of (16) with resource constraints, under the MEF-TOD scheduling strategy, if there are symmetric positive matrices $W, Q$, and $S$, an ordinary matrix $K_{\sigma}$ (where $\sigma \in\{1,2, \cdots, N\}$ is the corresponding data transmission pattern under the scheduling protocol), and a positive real number $\varepsilon_{\sigma}$, making linear matrix inequality (LMI) in (24) true, then the sliding mode motion defined by (21) of the closed-loop system Eq. (16) will create asymptotic stability on the sliding mode surface of (18), and it will represent an upper bound for the system performance indicator as

$$
\begin{aligned}
& J<x(0)^{T} W x(0)+\omega(-1)^{T} Q \omega(-1)+v(-1)^{T} S v(-1) \\
& {\left[\begin{array}{ccccccccccc}
-\bar{W} & 0 & 0 & \bar{W} \Xi_{1}^{T} & \bar{W} \Gamma_{\sigma}^{T} & \bar{W} \Xi_{2}^{T} & \bar{W} & \bar{W} \Xi_{3}^{T} & \bar{W} \Xi_{4}^{T} & 0 & \bar{W} \Gamma_{\sigma}^{T} \\
* & -\bar{Q} & 0 & 0 & \bar{Q}\left(I-\Gamma_{\sigma}\right)^{T} & 0 & 0 & 0 & 0 & 0 & \bar{Q}\left(I-\Gamma_{\sigma}\right)^{T} \\
* & * & -\bar{S} & \bar{S} \Xi_{5}^{T} & 0 & \bar{S}\left(I-\Delta_{\sigma}\right)^{T} & 0 & 0 & -\bar{S}\left(E \Delta_{\sigma}\right)^{T} & 0 & 0 \\
* & * & * & \Xi_{6}^{T} & 0 & 0 & 0 & 0 & 0 & \Xi_{7}^{T} & 0 \\
* & * & * & * & -\bar{Q} & 0 & 0 & 0 & 0 & 0 & 0 \\
* & * & * & * & * & -\bar{S} & 0 & 0 & 0 & \Delta_{\sigma} K_{\sigma} & 0 \\
* & * & * & * & * & * & -R_{1}^{-1} & 0 & 0 & 0 & 0 \\
* & * & * & * & * & * & * & -R_{2}^{-1} & 0 & K_{\sigma} & 0 \\
* & * & * & * & * & * & * & * & -\varepsilon_{\sigma} I & E \Delta_{\sigma} K_{\sigma} & 0 \\
* & * & * & * & * & * & * & * & * & -I & 0 \\
* & * & * & * & * & * & * & * & * & * & -I
\end{array}\right]}
\end{aligned}
$$

where

$$
\begin{aligned}
& \Xi_{1}=A-\left(A_{c}^{-1}(H-I)\right) \Delta_{\sigma}\left(L_{\sigma} B_{0}\right)^{-1} L_{\sigma} \Gamma_{\sigma} A, \\
& \Xi_{2}=-\Delta_{\sigma}\left(L_{\sigma} B_{0}\right)^{-1} L_{\sigma} \Gamma_{\sigma} A, \\
& \Xi_{3}=-\left(L_{\sigma} B_{0}\right)^{-1} L_{\sigma} \Gamma_{\sigma} A, \\
& \Xi_{4}=-E \Delta_{\sigma}\left(L_{\sigma} B_{0}\right)^{-1} L_{\sigma} \Gamma_{\sigma} A, \\
& \Xi_{5}=A_{c}^{-1}\left((A-I)-(H-I) \Delta_{\sigma}\right), \\
& \Xi_{6}=-W^{-1}+\varepsilon_{\sigma} \beta^{2} H H^{T}, \\
& \Xi_{7}=A_{c}^{-1}(H-I) \Delta_{\sigma} K_{\sigma}, \\
& \bar{W}=W^{-1}
\end{aligned}
$$

$$
\begin{aligned}
& \bar{Q}=Q^{-1}, \\
& \bar{S}=S^{-1} .
\end{aligned}
$$

Proof. The Lyapunov function is defined as

$$
\begin{aligned}
V(k)= & x(k)^{T} W x(k)+\omega(k-1)^{T} Q \omega(k-1) \\
& +v(k-1)^{T} S v(k-1)
\end{aligned}
$$

where variable $\xi(k)=\left[\begin{array}{lll}x^{T}(k) & \omega^{T}(k-1) & v^{T}(k-1)\end{array}\right]^{T}$ is defined, so the difference-equation model of $V(k)$ can be obtained by the system of (21) as 


$$
\begin{aligned}
& \Delta V(k)=V(k+1)-V(k)=\xi^{T}(k) \\
& \left\{\left[\begin{array}{c}
\Pi_{1 \sigma}^{T} \\
\Pi_{2 \sigma}^{T} \\
\Pi_{3 \sigma}^{T}
\end{array}\right] W\left[\begin{array}{c}
\Pi_{1 \sigma}^{T} \\
\Pi_{2 \sigma}^{T} \\
\Pi_{3 \sigma}^{T}
\end{array}\right]^{T}\right. \\
& +\left[\begin{array}{c}
\Gamma_{\sigma}^{T} \\
\left(I-\Gamma_{\sigma}\right)^{T} \\
0
\end{array}\right] Q\left[\begin{array}{c}
\Gamma_{\sigma}^{T} \\
\left(I-\Gamma_{\sigma}\right)^{T} \\
0
\end{array}\right]^{T} \\
& +\left[\begin{array}{c}
\Lambda_{1 \sigma}^{T} \\
\Lambda_{2 \sigma}^{T} \\
\left(I-\Delta_{\sigma}\right)^{T}
\end{array}\right] S\left[\begin{array}{c}
\Lambda_{1 \sigma}^{T} \\
\Lambda_{2 \sigma}^{T} \\
\left(I-\Delta_{\sigma}\right)^{T}
\end{array}\right]^{T} \\
& +\left[\begin{array}{c}
\Omega^{T} \\
\left(K_{\sigma}\left(I-\Gamma_{\sigma}\right)\right)^{T} \\
0
\end{array}\right] R_{2}\left[\begin{array}{c}
\Omega^{T} \\
\left(K_{\sigma}\left(I-\Gamma_{\sigma}\right)\right)^{T} \\
0
\end{array}\right]^{T} \\
& \left.+\left[\begin{array}{ccc}
R_{1}-W & 0 & 0 \\
0 & -Q & 0 \\
0 & 0 & -S
\end{array}\right]\right\} \xi(k)-\left[x^{T}(k) R_{1} x(k)\right. \\
& \left.+u^{T}(k) R_{2} u(k)\right]=\xi^{T}(k) \Psi \xi(k) \\
& -\left[x^{T}(k) R_{1} x(k)+u^{T}(k) R_{2} u(k)\right]
\end{aligned}
$$

where

$$
\begin{aligned}
& \Pi_{1 \sigma}^{T}=A+M_{0} \Delta_{\sigma} K_{\sigma} \Gamma_{\sigma}-M_{0} \Delta_{\sigma}\left(L_{\sigma} B_{0}\right)^{-1} L_{\sigma} \Gamma_{\sigma} A, \\
& \Pi_{2 \sigma}^{T}=M_{0} \Delta_{\sigma} K_{\sigma}\left(I-\Gamma_{\sigma}\right),
\end{aligned}
$$

$$
\begin{aligned}
\Pi_{3 \sigma}^{T} & =M_{0}\left(I-\Delta_{\sigma}\right)+M_{1}, \\
\Lambda_{1 \sigma}^{T} & =\Delta_{\sigma}\left(K_{\sigma} \Gamma_{\sigma}-\left(L_{\sigma} B_{0}\right)^{-1} L_{\sigma} \Gamma_{\sigma} A\right), \\
\Lambda_{2 \sigma}^{T} & =\Delta_{\sigma} K_{\sigma}\left(I-\Gamma_{\sigma}\right), \\
\Omega & =K_{\sigma} \Gamma_{\sigma}-\left(L_{\sigma} B_{0}\right)^{-1} L_{\sigma} \Gamma_{\sigma} A .
\end{aligned}
$$

According to (27), while $\Psi<0$, then $\Delta V(k)<0$ is true, so the system sliding mode motion defined by (21) can be ensured to be asymptotically stable, and the following relationship can be obtained from (27).

$$
x(k)^{T} R_{1} x(k)+u(k)^{T} R_{2} u(k)<-\Delta V(k)
$$

When summing (29) from $k=0$ to $k=+\infty$ and considering the system's (see (21)) asymptotic stability, which is supposed to be $V(+\infty)=0$, then

$$
\begin{aligned}
J< & V(0) \\
= & x(0)^{T} W x(0)+\omega(-1)^{T} Q \omega(-1) \\
& +v(-1)^{T} S v(-1)
\end{aligned}
$$

So the system cost function $J$ has an upper bound.

According to Schur's complement lemma, $\Theta<0$ is equivalent to

$$
\left[\begin{array}{ccccccc}
R_{1}-W & 0 & 0 & \Pi_{1 \sigma}^{T} & \Gamma_{\sigma}^{T} & \Lambda_{1 \sigma}^{T} & \Omega^{T} \\
* & -Q & 0 & \Pi_{2 \sigma}^{T} & \left(I-\Gamma_{\sigma}\right)^{T} & \Lambda_{2 \sigma}^{T} & \left(K_{\sigma}\left(I-\Gamma_{\sigma}\right)\right)^{T} \\
* & * & -S & \Pi_{3 \sigma}^{T} & 0 & \left(I-\Delta_{\sigma}\right)^{T} & 0 \\
* & * & * & -W^{-1} & 0 & 0 & 0 \\
* & * & * & * & -Q^{-1} & 0 & 0 \\
* & * & * & * & * & -S^{-1} & 0 \\
* & * & * & * & * & * & -R_{2}^{-1}
\end{array}\right]<0
$$

Then according to the definitions of parameters $\Pi_{i \sigma}(i=$ $1,2,3), \Lambda_{j \sigma}(j=1,2,3)$, and $\Omega$, with the adoption lemma and
Schur's complement lemma, the following can be obtained by the equivalent transform of (31). 


$$
\left[\begin{array}{ccccccccccc}
-W & 0 & 0 & \Xi_{1}^{T} & \Gamma_{\sigma}^{T} & \Xi_{2}^{T} & I & \Xi_{3}^{T} & \Xi_{4}^{T} & 0 & \Gamma_{\sigma}^{T} \\
* & -Q & 0 & 0 & \left(I-\Gamma_{\sigma}\right)^{T} & 0 & 0 & 0 & 0 & 0 & \left(I-\Gamma_{\sigma}\right)^{T} \\
* & * & -S & \Xi_{5}^{T} & 0 & \left(I-\Delta_{\sigma}\right)^{T} & 0 & 0 & \left(-E \Delta_{\sigma}\right)^{T} & 0 & 0 \\
* & * & * & \Xi_{6}^{T} & 0 & 0 & 0 & 0 & 0 & \Xi_{7}^{T} & 0 \\
* & * & * & * & -Q^{-1} & 0 & 0 & 0 & 0 & 0 & 0 \\
* & * & * & * & * & -S^{-1} & 0 & 0 & 0 & \Delta_{\sigma} K_{\sigma} & 0 \\
* & * & * & * & * & * & -R_{1}^{-1} & 0 & 0 & 0 & 0 \\
* & * & * & * & * & * & * & -R_{2}^{-1} & 0 & K_{\sigma} & 0 \\
* & * & * & * & * & * & * & * & -\varepsilon_{\sigma} I & E \Delta_{\sigma} K_{\sigma} & 0 \\
* & * & * & * & * & * & * & * & * & -I & 0 \\
* & * & * & * & * & * & * & * & * & * & -I
\end{array}\right]<0
$$

For convenient solution, $\bar{W}=W^{-1}, \bar{Q}=Q^{-1}$, and $\bar{S}=S^{-1}$ are defined, so the LMI (see (24)) can be obtained by the left and right multiplication of $\operatorname{diag}(\bar{W}, \bar{Q}, \bar{S}, I, I, I, I, I, I, I, I)$ to (32), which completes the proof.

3.3. Heuristic Joint Optimization for Sliding Mode Surface Gain and Network Parameters. According to the parameter definitions in (12), the influence of network parameters on system performance is reflected in the changes of the model coefficient matrices, so the corresponding sliding mode surface gain matrix of some network parameter combination can be obtained by Theorem 5 . However, in consideration of the joint design of two parameters, the problem above should be converted into a performance function optimization based on inequality constraints. Let the initial system state be $\xi(0)=$ $\left[x(0)^{T} \omega(-1)^{T} v(-1)^{T}\right]^{T}$, so the joint optimization study for sliding mode surface gain and network parameters can be obtained as

$$
\begin{array}{cl}
\min _{W, Q, S} & {\left[x(0)^{T} W x(0)+w(-1)^{T} Q w(-1)+v(-1)^{T} S v(-1)\right]} \\
\text { s.t. } & \operatorname{LMI}(24), \quad \sigma \in\{1,2, \cdots, N\} \\
& \varepsilon_{\sigma}>0 \\
& W>0 \\
& Q>0 \\
& S>0 \\
& \frac{\iota P}{\eta}<T<T_{\max }, \quad P \in\{1,2, \cdots, n+m\}
\end{array}
$$

Because of the nonlinear mapping relationship between LMI (see (24)) and network parameters $T$ and $P$, the optimization problem given by (33) cannot be solved directly. Focusing on this problem, a heuristic joint design method is proposed as follows.

Step 1. Sliding mode surface gain matrix $L_{\sigma}$ is defined to make $L_{\sigma} B_{0}$ nonsingular. Let the initial data packet capacity be $P_{0}=1$, sampling period be $T_{0}=\iota P_{0} / \eta$, and sampling period search step length be $h=1 \mathrm{~ms}$.

Step 2. By submitting network parameter $\left\{P_{0}, T_{0}\right\}$ into the optimization problem of (33), a feasible solution $\left\{W, Q, S, \varepsilon_{\sigma}\right.$,
$\left.K_{\sigma}\right\}$ can be obtained, and performance function upper bound $J_{0}^{*}$ can be calculated; If the optimization problem has no solution, the sampling period should be updated as $T=T_{0}+$ $h \times i,\left(i \in Z^{+}\right)$, and then substitute $\left\{P_{0}, T\right\}$ into (33) and repeat the calculation until the optimization solvable.

Step 3. Based on Step 2, a new performance function upper bound $J_{1}^{*}$ should be calculated with $T=T_{0}+h$, and if $J_{1}^{*}<$ $J_{0}^{*}$, the calculation should be repeated until $J_{i+1}^{*}>J_{i}^{*}(i$ is the solver iteration count for the optimization), so $J_{(0)}^{*}=J_{i}^{*}$ will be the minimum performance upper bound under the condition of data packet capacity $P=P_{0}$, and $T$ will be the optimal sampling period for $P$. 
Step 4. After $P=P_{0}+j,(j=1,2, \cdots, n+m-1)$ is defined, Step 1-Step 3 should be repeated, creating a parameter combination space $\left\{P_{j}, T_{j}, J_{(j)}^{*}\right\}$, and then every upper bound obtained should be compared to the minimum $J_{(j)}^{*}$ and the corresponding $P_{j}, T_{j}$. Finally, the optimal sliding mode surface gain $K_{\sigma}$ can be calculated.

Compared to the previously assumed brute force enumerative method and grid search method, the computation efficiency is greatly improved by the heuristic algorithm. Although some intelligent optimizing methods may be better than the heuristic algorithm to reduce computational cost, the proposed method is simple, reliable, and of certain practical value in consideration of the potential no solution of the optimization problem. The number of times that the heuristic algorithm takes for the variable calculations is mainly influenced by $h$, and when $P$ has been set and $h=0.001 \mathrm{~s}$, the computational cost is approximately 40 times. There will be some variation if the corresponding two conditions have the same minimum upper bound $J_{(j)}^{*}$, and under this circumstance, the control qualities should be compared to determine the better network parameter combination.

\section{Design and Reachability Analysis for Piecewise Adaptive Sliding Mode Controller}

In ideal conditions, the higher the system sampling frequency, the better the real-time data of the system state and control information. However, with resource constraints, system sampling frequency and data packet capacity must have upper bounds. In reality, for both QoS and QoP, the real sampling frequency might be lower than the ordinary condition, which also means a larger sampling period $T$. The sampling period has more influence on the controller performance with respect to the standard discrete sliding mode control, which is reflected in the following: (1) System chattering amplitude will increase with larger sampling period $T$; (2) under the effect of scheduling, the system will abandon some data packets, so the system robustness will be influenced by the slower updating of state and control values. In this section, as the sampling period is variable with a discrete reaching law with adaptive adjustment capability, the piecewise adaptive sliding mode controller is designed mainly for reducing chattering, enhancing system robustness, and ensuring the system reaches quasi-sliding mode motion in limited time.

4.1. Piecewise Adaptive Reaching Law Design Based on Attenuation Factor. In consideration of the time-varying characteristic of the sliding mode surface function, variables are defined as

$$
\begin{aligned}
& p=\alpha_{1}\|s(k)\|^{\gamma_{1}} \\
& \varsigma=\alpha_{2}\|s(k)\|^{\gamma_{2}}
\end{aligned}
$$

where $\alpha_{1}, \alpha_{2}>0, \gamma_{1}, \gamma_{2}>1$. The adaptive exponential reaching law is defined as

$$
s(k+1)-s(k)=-p T S(k)-\varsigma T \operatorname{sgn}(s(k))
$$

From (35), when the distance between the system and sliding mode surface is longer, $p$ and $\varsigma$ should be larger to ensure the system reaches the sliding mode surface faster; when the system is close to the sliding mode surface, $p$ and $\varsigma$ should be smaller along with $\|s(k)\|$ to decrease the thickness of the sliding mode switching plane and to avoid system chattering. However, for better reachability of the sliding motion, the adaptive exponential reaching law of (35) should satisfy the condition of sampling time as follows [23]:

$$
T<\frac{2}{\left(\alpha_{1}+\alpha_{2}\right)|s(0)|}
$$

With network scheduling, the value $T$ might be larger outside the upper bound of (36) based on the joint design method discussed in Section 3.

In fact, during the reaching process, the traditional exponential reaching law less-strict requirements on the sampling period may significantly increase the reaching speed. However, when the system reaches the quasi-sliding mode motion, larger chattering will be expressed. The reaching law is designed by $s(k+1)=s(k)$ for the traditional exponential reaching law, but if the system is reaching, the sliding controller should draw the system state into the motion of sliding mode, so [24]

$$
\|s(k+1)\|<\|s(k)\|
$$

An attenuation factor should be considered for sliding mode surface function, and the reaching relationship obtained is

$$
s(k+1)=\phi s(k)
$$

where $0<\phi<1$ is the attenuation factor which should be designed.

Given the above, this section proposes an adaptive discrete sliding mode reaching law based on an attenuation factor to improve the dynamic performance of sliding mode control, which is defined as

$$
\begin{aligned}
s(k+1) & \\
& = \begin{cases}\phi s(k) & \|s(k)\| \geq \mu \\
(1-p T) s(k)-\varsigma T \operatorname{sgn}(s(k)) & \|s(k)\|<\mu\end{cases}
\end{aligned}
$$

where $\mu>0$ is the given reaching law switching bound.

4.2. Reachability Analysis of Sliding Mode Controller. By substituting (20) into (19), the following can be obtained from (16).

$$
s(k+1)=L_{\sigma} \Gamma_{\sigma} \Delta B \Delta v(k)=r(k)
$$

where $\Delta v(k)=v(k)-v(k-1)$, and $r(k)$ is the control residual under the influence of $u_{e q}(k)$.

According to (20), (39), and (40), the sliding mode control law is presented as 


$$
\begin{aligned}
& u(k)= \begin{cases}u_{e q}(k)+\left(L_{\sigma} B_{0}\right)^{-1}(\phi s(k)-\widetilde{r}(k)) & \|s(k)\| \geq \mu \\
u_{e q}(k)+\left(L_{\sigma} B_{0}\right)^{-1}[(1-p T) s(k)-(\varsigma T+\chi) \operatorname{sgn}(s(k))]-\left(L_{\sigma} B_{0}\right)^{-1} \tilde{r}(k) & \|s(k)\|<\mu\end{cases} \\
& \tilde{r}(k)= \begin{cases}\widetilde{r}(k-1)+[s(k)-\phi s(k-1)] & \|s(k)\| \geq \mu \\
r(k-1) & \|s(k)\|<\mu\end{cases}
\end{aligned}
$$

where $u_{e q}(k)$ is defined the same as in $(20), \widetilde{r}(k)$ is designed to compensate for the influence of $r(k)$, and $\chi$ is the upper bound of the changing rate norms of $r(k)$; that is, when $\| r(k)-$ $r(k-1) \| \leq \chi, \mu=(1 /(1-\phi)) \chi$ is reaching the law switching boundary.

Theorem 6. When considering the networked control system with resource constraints of (16) and the linear discrete sliding mode surface of (18) is adopted, then under the control law of (41) the system can reach the sliding mode surface in a limited time and finally stabilizes in the neighborhood of the ideal sliding mode surface.

Proof. When $\|s(k)\| \geq \mu$, the following can be obtained by substituting (16) and (41) into (19).

$$
s(k+1)=r(k)-\widetilde{r}(k)+\phi s(k)
$$

From (43),

$$
s(k)-\phi s(k-1)=r(k-1)-\widetilde{r}(k-1)
$$

By substituting (44) into (42),

$$
\widetilde{r}(k)=\widetilde{r}(k-1)+[s(k)-\phi s(k-1)]=r(k-1)
$$

For any bounded $\widetilde{r}(0)$ and from (44) and (45),

$$
\begin{aligned}
& s(1)=\phi s(0)+(r(0)-\widetilde{r}(0)) \\
& s(2)=\phi s(1)+(r(1)-r(0))
\end{aligned}
$$

When $k>1$, it can be determined from (46) that

$$
\begin{aligned}
s(k)= & \phi^{k-1} s(1) \\
& +\sum_{i=0}^{k-2} \phi^{i}[r(k-2-j+1)-r(k-2-j)]
\end{aligned}
$$

The maximum changing rate of $r(k)$ is defined as $\| r(k)-r(k-$ $1) \|<\chi$, and the following can be obtained from (47).

$$
\begin{aligned}
\|s(k)\| \leq & \phi^{k-1}\|s(1)\| \\
& +\sum_{i=0}^{k-2} \phi^{i}\|r(k-2-j+1)-r(k-2-j)\| \\
= & \phi^{k-1}\|s(1)\|+\sum_{i=0}^{k-2} \phi^{i} \chi
\end{aligned}
$$

As $0<\phi<1$, when $k \longrightarrow \infty$,

$$
\|s(k)\|<\frac{1}{1-\phi} \chi
$$

So system will finally reach the quasi-sliding mode motion neighborhood whose thickness is $\mu$; that is, $\|s(k)\|<\mu$.

When $\|s(k)\|<\mu$,

$$
\begin{aligned}
s(k+1)-s(k)= & r(k)-\tilde{r}(k)-\chi \operatorname{sgn}(s(k)) \\
& -q T s(k)-\varepsilon T \operatorname{sgn}(s(k))
\end{aligned}
$$

According to the definition of $\chi$, the relationships below are obtained:

$$
\begin{aligned}
& r(k)-\tilde{r}(k) \leq\|r(k)-\tilde{r}(k)\| \leq \chi \operatorname{sgn}(s(k)) \\
& s(k)>0 \\
& r(k)-\tilde{r}(k) \geq-\|r(k)-\tilde{r}(k)\| \geq \chi \operatorname{sgn}(s(k)) \\
& s(k)<0
\end{aligned}
$$

Then the following inequality can be obtained.

$$
\begin{gathered}
s(k+1)-s(k) \leq(1-p T) s(k)-\varsigma T \operatorname{sgn}(s(k)) \\
s(k)>0 \\
s(k+1)-s(k) \geq(1-p T) s(k)-\varsigma T \operatorname{sgn}(s(k)) \\
s(k)<0
\end{gathered}
$$

Given the above, under the effect of the control law of (41), the system will finally reach and stabilize in the stable neighborhood of the sliding mode surface.

So when $\|s(k)\| \geq \mu$ and there is no significant distance between the system and sliding mode surfaces, it can be ensured that system can reach stability faster after adopting the sliding mode control law based on an attenuation factor, which also avoids the influence of the sampling period on reachability; when $\|s(k)\|<\mu$, system chattering will be weakened after adopting the sliding mode control law based on an adaptive exponential reaching law.

\section{Simulation and Results Analysis}

5.1. TrueTime Simulation Platform for Aeroengine Networked Control Systems. To verify the sliding mode controller joint design method and study the influence of the scheduling 


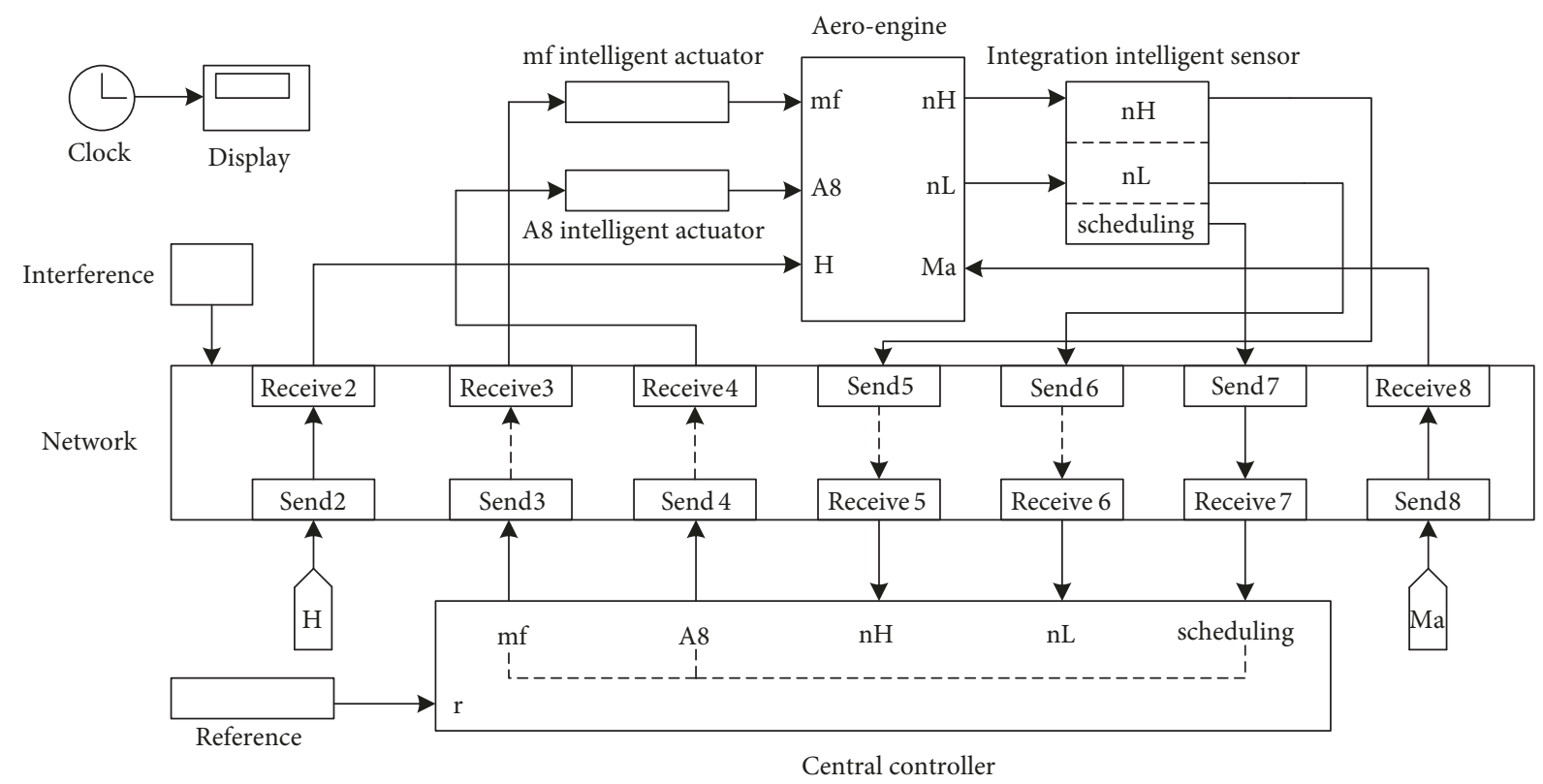

FIGURE 2: Simplified block model of the TrueTime simulation for an aeroengine networked control system.

strategy on the system, an aeroengine networked control system simulation platform is established based on the Matlab TrueTime/Simulink, whose simplified block model is shown in Figure 2. A small deviation state space model of some type of aeroengine is chosen as the study object for several under-fixed conditions [25], and with an independent module (aeroengine) encapsulated by Simulink the dynamic characteristics of the corresponding engine steadystate points are simulated. As model requirements for input, output, and scheduling, one integration intelligent sensor node $\left(n_{H}, n_{L}\right.$ sensor), two intelligent actuator nodes $\left(m_{f}, A_{8}\right.$ actuator), one central controller node (central controller), one communication network module (network), and one nonperiodic interference node (Interference) are set.

Sensor and actuator nodes are established by the True Time Kernel in the TrueTime toolbox, which includes a flexible real-time kernel to transform $\mathrm{A} / \mathrm{D}$ and D/A and to transmit periodic and nonperiodic data. For each sensor or actuator, the node initialization and node mission functions are most important and are used to definite external interface quantities of nodes, access priority, sampling period, data processing time delay, etc. Furthermore, the MEF-TOD scheduling module is designed in the sensor node, which receives state messages sent by the engine. By comparing the data with the control input from the last sampling period, the transmission pattern in the current period can be calculated by (7) and (8). The central controller node is also established by True Time Kernel, which will provide sliding control signal $u$ and switching function $s$. The nonperiodic interference node can influence network load to simulate some conditions like time-varying delays, transmission collisions, etc. by changing network shared parameters. The communication network module is established by TrueTime Network, and based on message priority arbitration a CAN bus network model with Carrier Sense Multiple Access (CSMA/AMP) is adopted. Node access order is determined with a selfowned priority as exclusive identification in the bus network, and when the network is busy, any node message will wait until a quiet period. If there is a network access conflict, node messages with higher priority will be permitted to transmit. For the aeroengine nonlinear model, data interfaces and sender and receiver nodes of flight height $H$ and Mach number $M a$ are reserved in the simulation platform.

5.2. Joint Simulation for Network Parameters and Controller. The proposed control method is generalizable for aeroengine networked control systems based on small deviation state space models, which are widely used as basic models in aeroengine control research. On the condition of maximum rating on the ground, a continuous state space model of an aeroengine is adopted, and the specific parameters are

$$
\begin{aligned}
A_{c} & =\left[\begin{array}{cc}
-3.573 & 0.672 \\
0.337 & -3.492
\end{array}\right], \\
B_{c} & =\left[\begin{array}{ll}
0.496 & 0.669 \\
0.636 & 3.604
\end{array}\right], \\
C_{c} & =\left[\begin{array}{ll}
1 & 0 \\
0 & 1
\end{array}\right] \\
x & =\left[\begin{array}{l}
\Delta n_{H} \\
\Delta n_{L}
\end{array}\right] \\
u & =\left[\begin{array}{l}
\Delta m_{f} \\
\Delta A_{8}
\end{array}\right], \\
y & =\left[\begin{array}{l}
\Delta n_{H} \\
\Delta n_{L}
\end{array}\right]
\end{aligned}
$$


where $n_{L}$ is the low pressure rotor speed, $n_{H}$ is the high pressure rotor speed, $m_{f}$ is the amount of fuel for the main combustion chamber, and $A_{8}$ is the nozzle area. Let the CAN bus network bandwidth $\eta=30000 \mathrm{bit} / \mathrm{s}$, and data width $\iota=110$ during one transmission period under the CAN2.0 protocol standard. Non-real-time data comes from the interference node in the simulation platform with data sending probability of $20 \%$, and the data type is a random number within $[0,1]$ and data width $\theta_{k}=80$ bit. The unknown network parameters $T$ and $P$ meet the constraint of

$$
\theta_{k}+\iota P \leq T \eta
$$

The common sliding mode surface parameter matrix is $L_{\sigma}=$ $\operatorname{diag}(I, I)$. From (54), the constrained optimization problem defined by (33) can be solved by the method in Section 3.3, and the optimal network parameter combination $\{T, P\}=$ $\{0.015,3\}$ and the corresponding optimal performance function upper bound $J^{*}=2.3268$ can be determined. In the current sampling period, the system input coefficient matrix can be calculated by discretization as

$$
B_{0}=\left[\begin{array}{ll}
0.0037 & 0.0050 \\
0.0047 & 0.0267
\end{array}\right]
$$

It is easy to show that $L_{\sigma} B_{0}$ is invertible, and the four data transmission patterns and sliding mode surface parameters can be solved as

$$
\begin{aligned}
& \Psi_{1}=\operatorname{diag}(1,0,1,1), \\
& K_{1}=\left[\begin{array}{ll}
-0.1042 & -1.3674 \\
-0.0126 & -0.0921
\end{array}\right] \\
& \Psi_{2}=\operatorname{diag}(0,1,1,1), \\
& K_{2}=\left[\begin{array}{ll}
-0.0301 & -0.4193 \\
-0.0286 & -0.3709
\end{array}\right] \\
& \Psi_{3}=\operatorname{diag}(1,1,0,1), \\
& K_{3}=\left[\begin{array}{cc}
0.0190 & 0.1510 \\
-0.0136 & -0.1860
\end{array}\right] \\
& \Psi_{4}=\operatorname{diag}(1,1,1,0), \\
& K_{4}=\left[\begin{array}{cc}
0.0218 & 0.1808 \\
-0.0159 & -0.2253
\end{array}\right]
\end{aligned}
$$

The switching boundary of the sliding mode control law is defined as $\mu=0.2$, sliding mode surface attenuation factor is $\phi=0.6$, and the adaptive exponential reaching law parameters are $p=20, \varsigma=2, \gamma_{1}=\gamma_{2}=2, \chi=0.5$. To verify the system tracking effect under command signals, step responses of system states are studied. From (41), the sliding mode tracking controller is designed as

$$
u(k)= \begin{cases}u_{r}(k)+\left(L_{\sigma} B_{0}\right)^{-1}(\phi s(k)-\tilde{r}(k)) & \|s(k)\| \geq \mu \\ u_{r}(k)+\left(L_{\sigma} B_{0}\right)^{-1}[(1-p T) s(k)-(\varsigma T+\chi) \operatorname{sgn}(s(k))]-\left(L_{\sigma} B_{0}\right)^{-1} \tilde{r}(k) & \|s(k)\|<\mu\end{cases}
$$

where $u_{r}(k)=\left(L_{\sigma} B_{0}\right)^{-1} L_{\sigma} R(k+1)-u_{e q}(k)$.

For realistic simulation of the influence of network conditions on system performance, the packet dropping probability in CAN bus network is set as 0.1 , and a 1-ms sender time delay and preprocessing time delay exist in sensors and controllers. System step response results under the effects of the controller are shown in Figure 3, in which the red curves are effects of the sliding mode controller designed in this paper. From Figure 3, asymptotic stability for the system sliding mode motion with network constraints can be ensured by designing the sliding mode surface parameters according to Theorem 5 , which reduces the steady-state error of the system.

After adopting the piecewise adaptive sliding mode controller, the limitation of the sampling period on the sliding mode control law can be avoided. The system has ideal reaching motion performance, and overshoots do not exist. The blue curves show controller response results in [7] based on the controller state feedback design, and although asymptotic stability can also be ensured, overshoots are obvious because of the absence of optimization for system dynamic performance. The input signals have been provided in Figure 4, and the existing weak signal fluctuations have little influence on the output signals. The simulated actuator characteristic times, which can be modified for the real aeroengine actuators to follow, are set to be 0.05 ; that is, the proposed simulation method is reasonable when considering real actuator sensibility. Convergence curves of the sliding mode surface function are shown in Figure 5, which indicates that convergence to quasi-sliding mode motion over a shorter time can be ensured by adopting the piecewise adaptive reaching law method designed in this paper. From the magnification in Figure 5, when the system switches to the adaptive sliding mode reaching law, the reaching law coefficient converges with $\|s(k)\|$, and system chattering is reduced.

In consideration of the presence of disturbances and random packet-loss, by using the interference node to send noise to itself, five conditions are considered with $10 \%, 30 \%$, $50 \%, 70 \%$, and $90 \%$ of the network bandwidth occupied by the disturbances; moreover, a random packet-loss possibility is designed and introduced into the simulation after $2 \mathrm{~s}$, and the results are shown in Figure 6. According to Figure 6, good reachability is demonstrated, and with increased disturbance, the system stability is initially weakened but then regains 


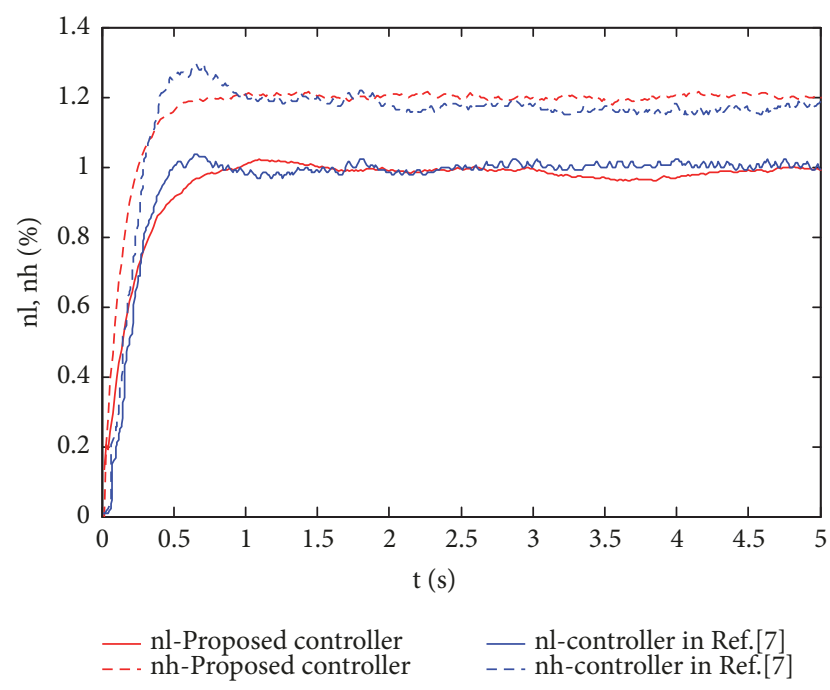

FIgURE 3: Step response results under control $(T=0.015 \mathrm{~s}, P=3)$.

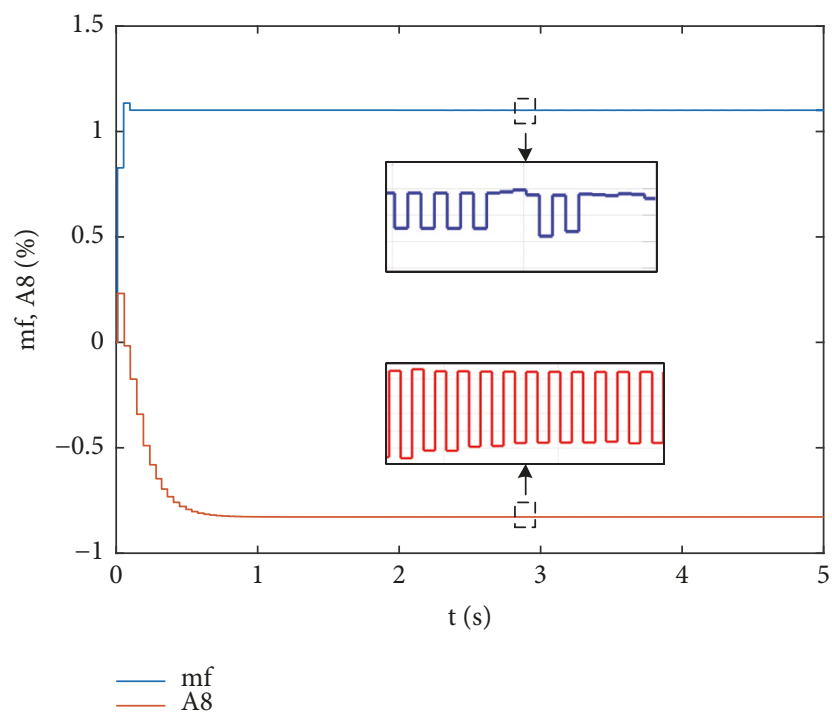

FIGURE 4: Input signals for the aeroengine model ( $T=0.015 \mathrm{~s}, P=3)$.

strength. Under the conditions of 70\% and 90\% bandwidth disturbance, the reason for the better system stabilities is the lower transmission of control signals; however, with random packet-loss possibility also existing in the actuators after $2 \mathrm{~s}$, better stabilities cannot be maintained. Thus, in the comparison of the overall reachability and stability, the results with $10 \%$ and $30 \%$ bandwidth disturbance show the effectiveness of the proposed approach.

As $P$ is varied from 1 to 4 , when $P=4$, all signals will be sent, so the scheduling strategy cannot be verified and analyzed. When $P=1$, the sliding mode controlling characteristics will be worse and the convergence of state values will be time-consuming, so $P=1$ cannot be the reference condition. Thus, when $P=2$, the best $T$ is 0.008 which is obtained by the heuristic joint optimization. After satisfying (33), another network parameter combination $\left\{T^{\prime}, P^{\prime}\right\}=\{0.008,2\}$ is adopted, and the corresponding step response results are

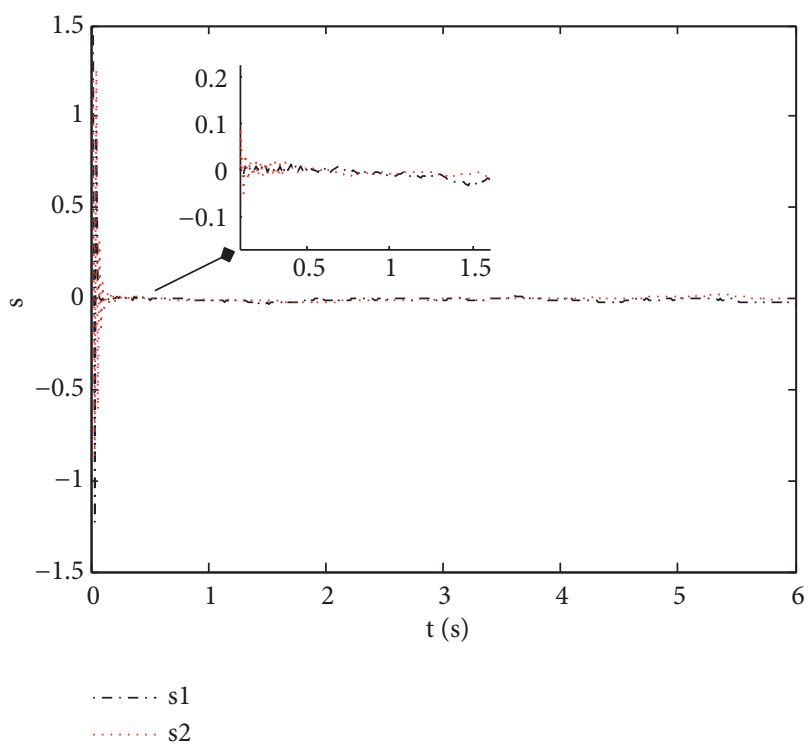

FIGURE 5: Convergence curves of sliding mode surface function.

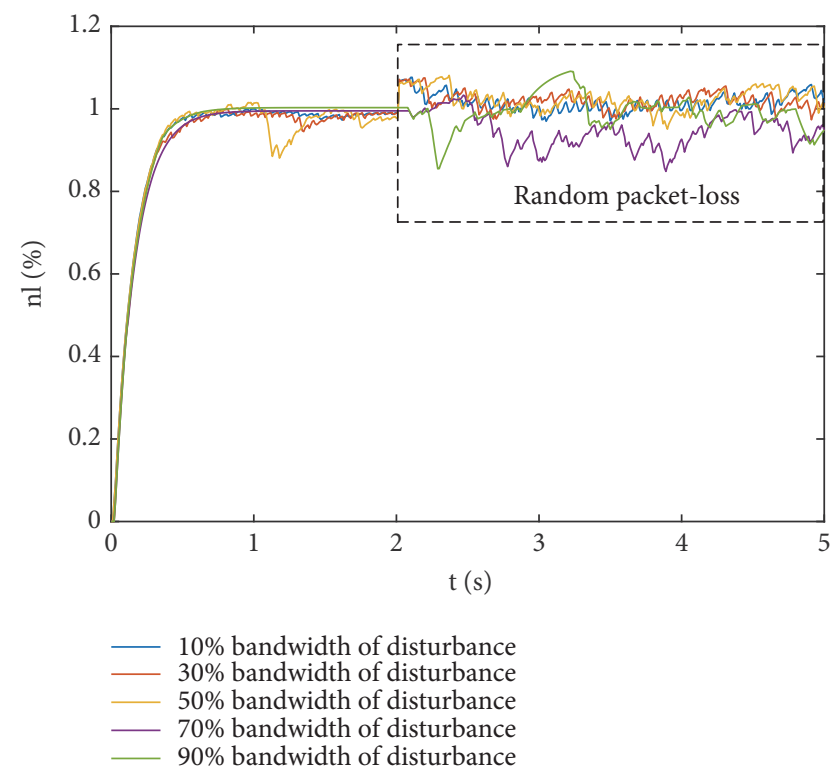

FIGURE 6: Simulation results with the presence of disturbances and random packet-loss.

shown in Figure 7. From the figure, there exist obvious steadystate errors and a decrease in system dynamic performance because optimization of the performance function $J$ by the joint designed sliding mode surface parameters cannot be ensured under the current network condition. With limitation of network bandwidth, higher data transmission frequency can only be realized by decreasing data packet capacity, so the control system data cannot be updated in timely fashion, and QoP will be influenced greatly.

Partial scheduling results under the two network conditions are shown in Figures 8 and 9, and from bottom to top, $m_{f}$ and $A_{8}$ are controller input scheduling curves, and $n_{H}$ and $n_{L}$ are state value scheduling curves. For every curve, the low 


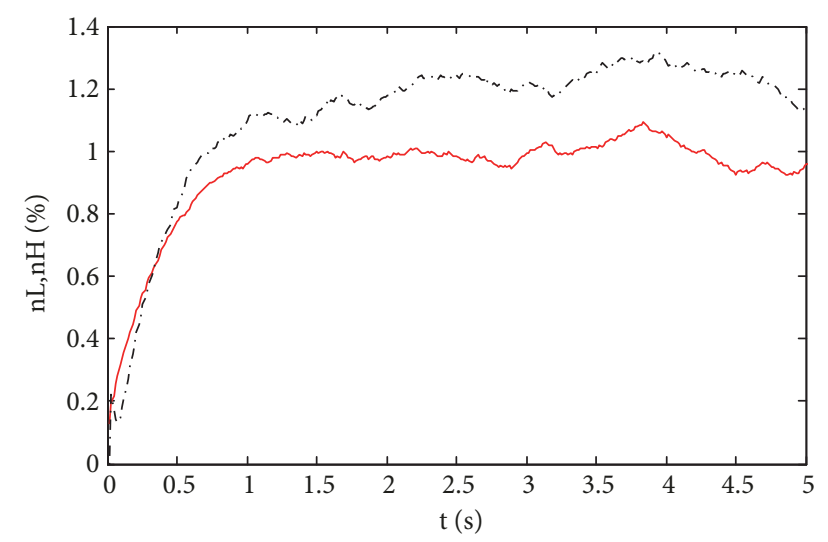

$-\mathrm{nL}$

Figure 7: Step response results under control $(T=0.008 \mathrm{~s}, P=2)$.

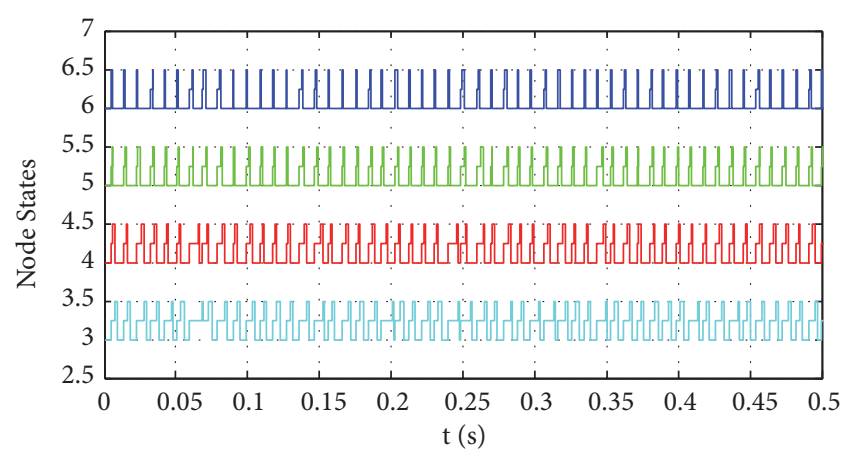

Figure 8: Network scheduling results $(T=0.015 \mathrm{~s}, P=3)$.

state represents "unsent", middle state represents "waiting to send", and high state represents "sending". From the figures, with the optimal network parameters $\{T, P\}=\{0.015,3\}$, the system network utilization is better than the previous parameter combination, and the data will be updated more quickly, so the simulation results in Figure 3 can be verified.

\section{Conclusion}

In this paper, for one type of aeroengine networked control system with resource constraints, a joint design method for network parameters and sliding mode control based on piecewise adaptive reaching law is presented. The joint model of the network parameters and control system is established for sliding mode control parameter analysis based on the MEFTOD scheduling strategy. In consideration of the influence of scheduling strategy, the asymptotic stability condition of sliding mode motion with a form of LMI is derived, and heuristic parameter optimization is considered to include the influence of network parameters. Finally, to avoid the influence of large sampling periods on the controller reaching motion performance, a piecewise adaptive sliding mode controller is designed to decrease system chattering based on an attenuation factor. By theoretical derivation and simulation analysis, the following conclusions are reached.

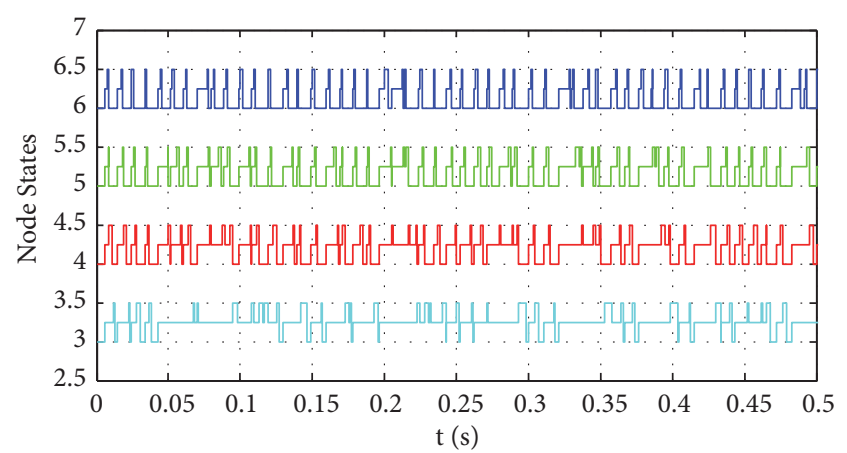

FIGURE 9: Network scheduling results $(T=0.008 \mathrm{~s}, P=2)$.

(1) When considering scheduling strategy, control system node data may not be updated in timely fashion during every sampling period. However, by joint modeling of network parameters and the control system, active packet dropping caused by the scheduling strategy can be reflected in the system model. Joint analysis allows design of a controller that considers the influence of sampling period $T$ and data packet capacity $P$ to enhance system performance.

(2) For the active packet dropping problem caused by network scheduling, the discrete sliding mode surface with compensation function, sliding mode surface gain, and network parameters are designed to ensure asymptotic stability of the system sliding mode motion. By heuristic optimization of network parameters and control system performance, a network parameter combination with the minimal performance function upper bound is obtained. The simulation results indicate that the designed sliding mode surface gain has ideal stabilization capability, and the closed-loop system has higher bandwidth utilization.

(3) Because of the bandwidth limitation in a communication network, system sampling frequency may not be high enough, so the corresponding sampling period $T$ will be larger. If the adaptive discrete reaching law is adopted, it will consider the sampling period to choose a better reaching law parameter. Therefore, an adaptive sliding mode reaching law is obtained based on attenuation factor, and then a piecewise adaptive sliding mode controller is designed. When the system is reaching, a higher speed to the sliding mode surface is ensured by the control law based on an attenuation factor. When system has reached to its limit, by switching into the adaptive sliding mode control law state convergence is ensured and chattering is effectively avoided. From the simulation results for the piecewise adaptive sliding mode controller designed in this paper, good dynamic performance and stronger robustness to active packet dropping are achieved.

(4) To extend this work to time-variant systems and nonlinear systems, some transition-state methods, such as the Takagi-Sugeno (T-S) fuzzy method, are necessary to connect and consider several different small deviation state space time-invariant models. In addition, the design and simulation methods of sensor and controller scheduling modules provide a new method to evaluate other nonlinear systems without small deviation state space model, such as 
intelligent control systems, by considering both controller and network performance.

\section{Data Availability}

The data used to support the findings of this study are available from the corresponding author upon request.

\section{Conflicts of Interest}

The authors declare that they have no conflicts of interest.

\section{Acknowledgments}

This work is supported by the National Natural Science Foundation of China (51606219 and 51476187).

\section{References}

[1] T. Adedokun and S. Zein-Sabatto, "Distributed control system for turbine engine optimal operations," in Proceedings of the 2013 IEEE Southeastcon, pp. 1-6, IEEE, 2013.

[2] D. B. Dačić and D. Nešić, "Quadratic stabilization of linear networked control systems via simultaneous protocol and controller design," Automatica, vol. 43, no. 7, pp. 1145-1155, 2007.

[3] H. Y. Chen, Research on control and scheduling methods for networked control systems with resource constraints [Ph.D. thesis], Zhejiang University, 2008, http://cdmd.cnki.com.cn/Ariticle/ CDMD-10337-2009058020.htm.

[4] M. Tabbara and D. Nesic, "Input-output stability of networked control systems with stochastic protocols and channels," Institute of Electrical and Electronics Engineers Transactions on Automatic Control, vol. 53, no. 5, pp. 1160-1175, 2008.

[5] L. H. Zhou, T. K. Wang, and D. P. Xu, "Survey on co-design of scheduling and control in networked control system," Journal of North China Electric Power University, vol. 35, no. 1, pp. 30-36, 2008.

[6] S. Fu and H. J. Fang, "Fault detection for NCSs subject to quantization and random packet dropout," Journal of Wuhan University of Technology (Information \& Management Engineering), vol. 33, no. 4, pp. 536-540, 2011.

[7] L. Wang, S. S. Xie, Z. G. Miao et al., "Collaborative fault tolerant control for aero-engine distributed control system with network scheduling," Journal of Propulsion Technology, vol. 39, no. 5, pp. 1164-1170, 2018.

[8] X. Jiang, Q.-L. Han, S. Liu, and A. Xue, "A new $\mathrm{H}_{\infty}$ stabilization criterion for networked control systems," IEEE Transactions on Automatic Control, vol. 53, no. 4, pp. 1025-1032, 2008.

[9] C. Zhou, M. Du, and Q. Chen, "Co-design of dynamic scheduling and H-infinity control for networked control systems," Applied Mathematics and Computation, vol. 218, no. 21, pp. 10767-10775, 2012.

[10] X. C. Zhu, C. Zhou, and Q. W. Chen, "Model-based average dwell time scheduling and control for networked control system," Control Theory Applications, vol. 32, no. 1, pp. 86-92, 2015.

[11] C. Zhou, H. Lu, J. Ren, and Q. Chen, "Co-design of dynamic scheduling and quantized control for networked control systems," Journal of The Franklin Institute, vol. 352, no. 10, pp. 39884003, 2015.
[12] H. Rehbinder and M. Sanfridson, "Scheduling of a limited communication channel for optimal control," Automatica, vol. 40, no. 3, pp. 491-500, 2004.

[13] Q. K. Shao, L. Yu, L. L. Ou, and D. Zhang, "Co-design methodology for network-based control systems based on QoS," Acta Automatica Sinica, vol. 36, no. 9, pp. 1356-1360, 2010.

[14] X. P. Wang, C. Zhou, and Q. W. Chen, "Simultaneous design of dynamic scheduling and control for networked control systems with time delay and constraints," Journal of Central South University (Science and Technology), vol. 44, no. 1, pp. 109-114, 2013.

[15] X. R. He, Research on discrete-time sliding mode variable structure control theory and application for aero-engine [Ph.D. thesis], Air Force Engineering University, 2009.

[16] M. Kchaou, H. Gassara, and A. El-Hajjaji, "Adaptive sliding mode control for fuzzy singular systems with time delay and input nonlinearity," International Journal of Adaptive Control and Signal Processing, vol. 32, no. 3, pp. 464-479, 2018.

[17] B. Lian, Q. Zhang, and J. Li, "Sliding mode control and sampling rate strategy for Networked control systems with packet disordering via Markov chain prediction," ISA Transactions, vol. 83, pp. 1-12, 2018.

[18] T. Wang, C. Zhou, H. Lu et al., "Hybrid scheduling and quantized output feedback control for networked control systems," International Journal of Control Automation \& Systems, vol. 16, no. 1, pp. 197-206, 2018.

[19] Y. Tang, F. Du, and X. Fang, "Deadband scheduling in sensor node and controller node for wireless networked control systems," International Journal of Wireless Information Networks, vol. 25, no. 3, pp. 241-249, 2018.

[20] X.-M. Tang and J.-S. Yu, "Feedback scheduling of model-based networked control systems with flexible workload," International Journal of Automation and Computing, vol. 5, no. 4, pp. 389-394, 2008.

[21] G. C. Walsh, H. Ye, and L. G. Bushnell, "Stability analysis of networked control systems," IEEE Transactions on Control Systems Technology, vol. 10, no. 3, pp. 438-446, 2002.

[22] Y. N. Guo, Q. Y. Zhang, D. W. Gong, and J. H. Zhang, "Robust fault-tolerant control of networked control systems with timevarying delays," Control and Decision, vol. 23, no. 6, pp. 689-692, 2008.

[23] W. Zhang, Research on variable structure control theory and application for aero-engine [Ph.D. thesis], Air Force Engineering University, 2008.

[24] M.-C. Pai, "Discrete-time sliding mode control for robust tracking and model following of systems with state and input delays," Nonlinear Dynamics, vol. 76, no. 3, pp. 1769-1779, 2014.

[25] S. Q. Fan, Aero-Engine Control, vol. 2, Northwestern Polytechnical University Press, 2008. 


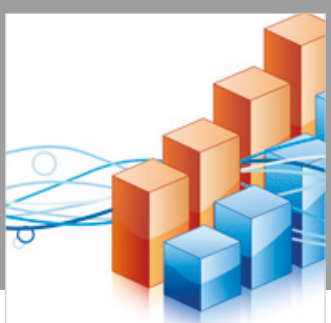

Advances in

Operations Research

\section{-n-m}
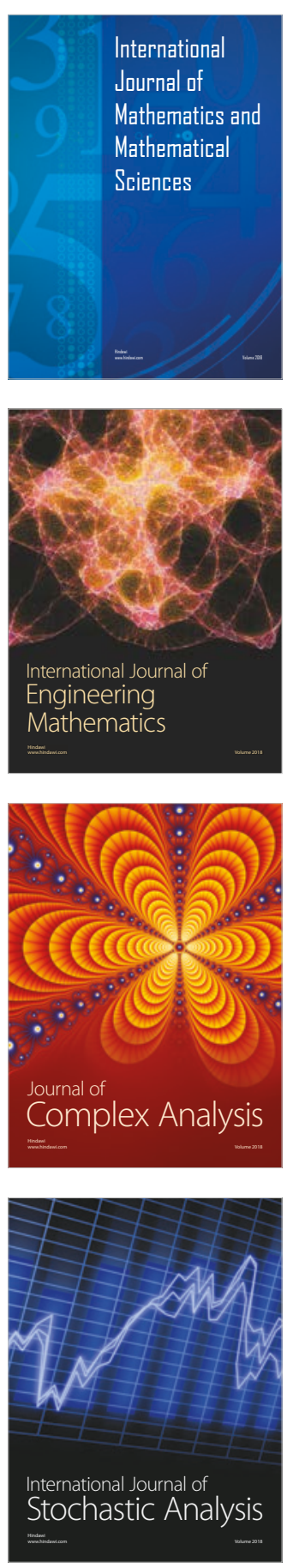
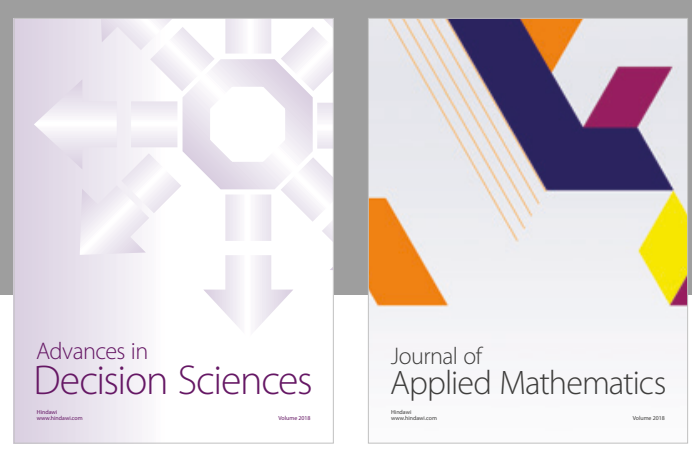

Journal of

Applied Mathematics
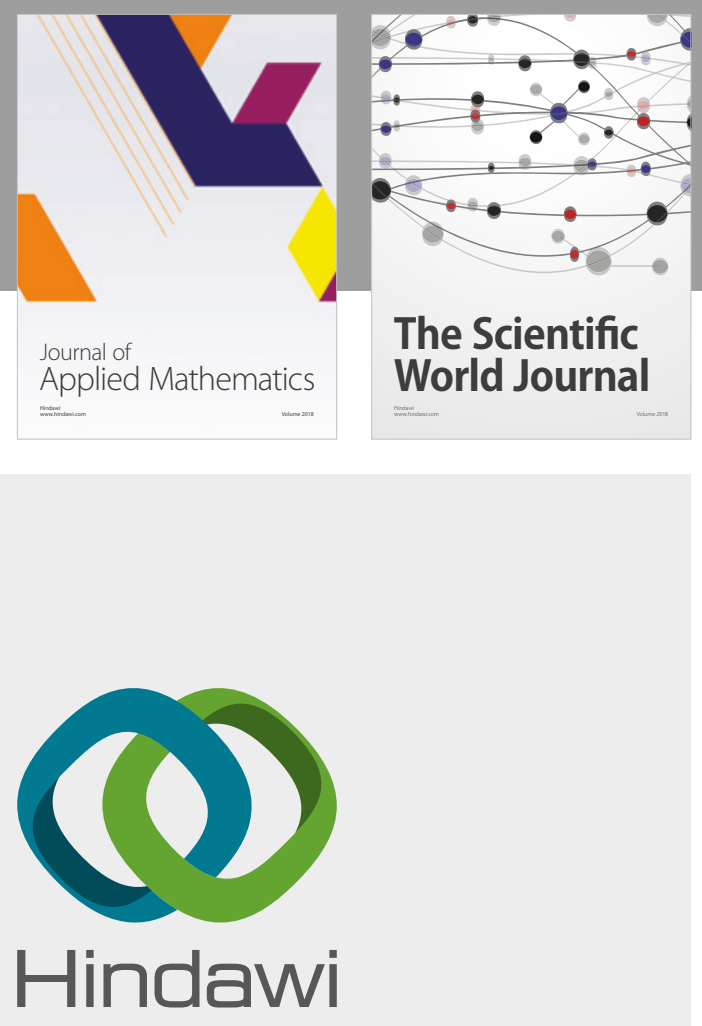

Submit your manuscripts at

www.hindawi.com

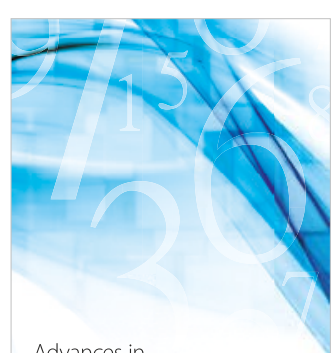

Advances in
Numerical Analysis
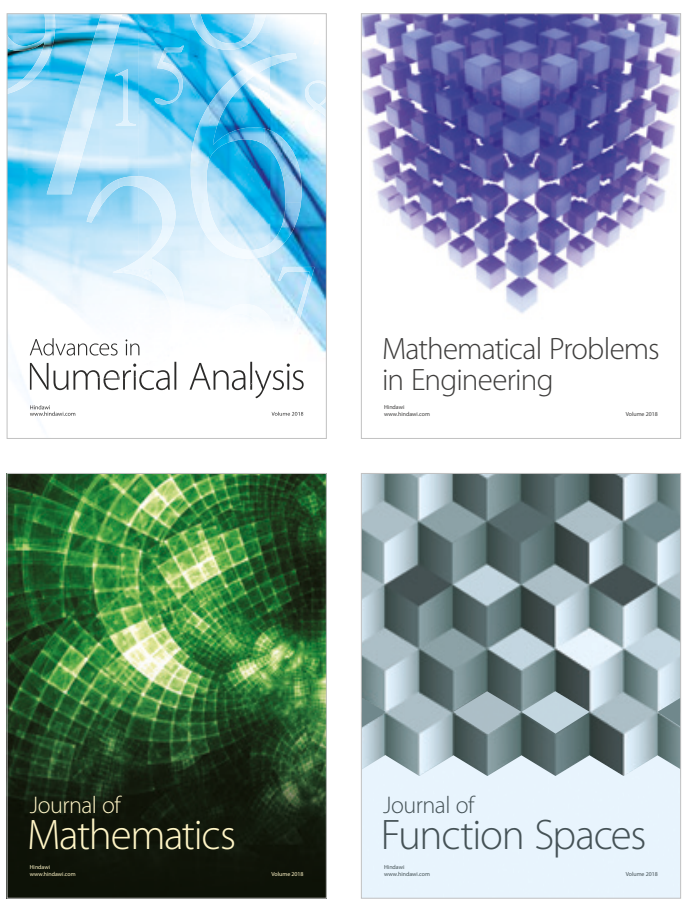

Mathematical Problems in Engineering

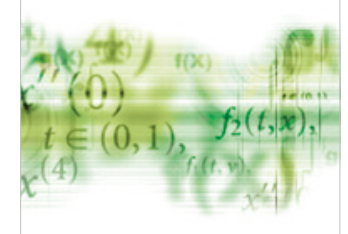

International Journal of

Differential Equations

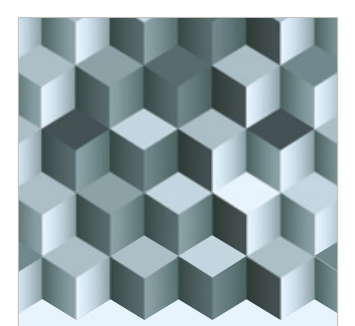

Journal of

Function Spaces
The Scientific

World Journal

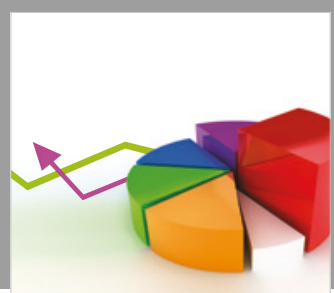

Journal of

Probability and Statistics
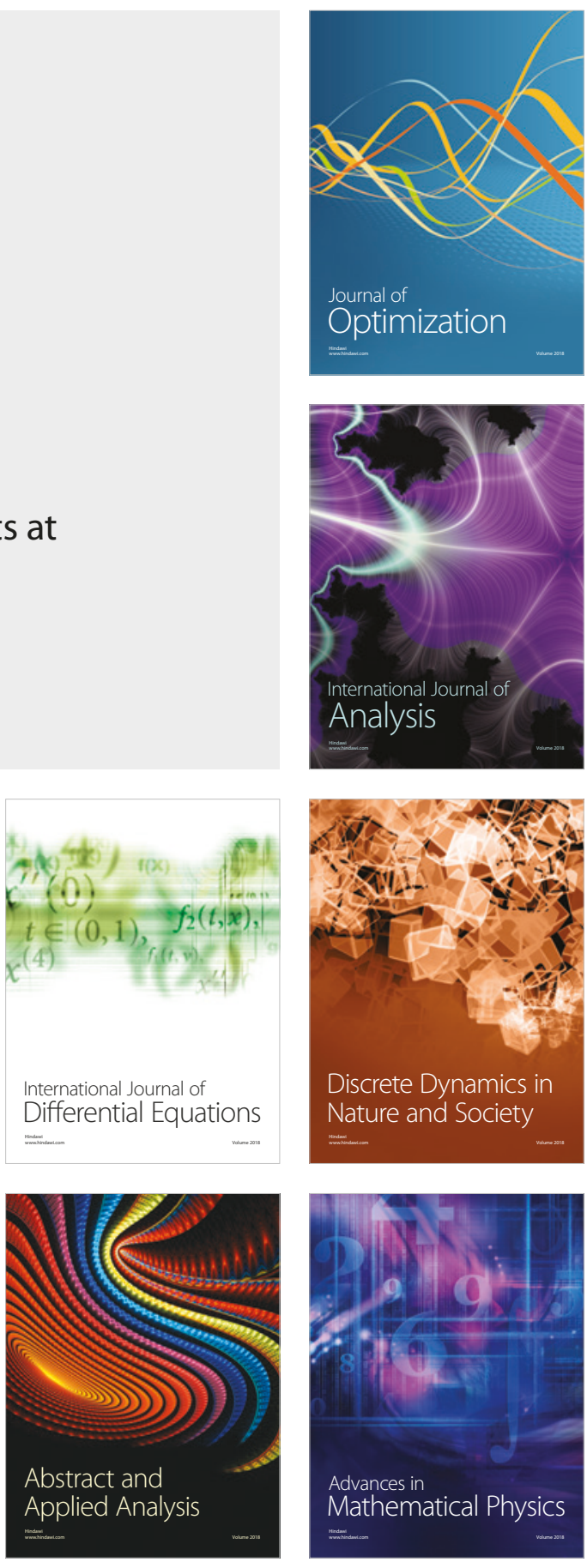